\title{
Verifying Duration Properties of Timed Transition Systems
}

Z. Liu

Department of Mathematics and Computer Science, University of Leicester, University Road, Leicester LE1 7RH, U.K.; E-mail: Z.Liu@mcs.le.ac.uk.

A. P. Ravn

Department of Information Technology, Technical University of Denmark, Building 344, Lyngby, Denmark; E-mail: apr@it.dtu.dk.

X. $\mathrm{Li}$

Faculty of Science and Technology, University of Macau, P.O. Box 3001, Macau; E-mail: fstxsl@umac.mo.

\begin{abstract}
This paper proposes a method for formal real-time systems development: Requirements and high level design decisions are time interval properties and are therefore specified in the Duration Calculus (DC), while implementations are described by timed transition systems (TTS). A link from implementation properties to the requirement and design properties is given by interpreting a DC formula in a model of the executions of a TTS and then providing rules for lifting properties proved by structural induction for a TTS to DC formulas. The method is illustrated by the Gas Burner case study.
\end{abstract}

\section{Keywords}

Real-time systems; Duration Calculus; Timed Transition Systems, Specification, Verification.

\section{INTRODUCTION}

Duration calculus (DC) was introduced to specify and reason about properties of embedded real-time systems (Zhou, Hoare \& Ravn 1991, Ravn, Rischel \& Hansen 1993), it has also been successfully used to reason about circuits and digital designs and hybrid systems (Ravn \& Rischel 1991, Zhou, Ravn \& Hansen 1993). Introductions to the applications are found in (Zhou 1993) while the logic and its foundation is given a thorough presentation in (Hansen \& Zhou 1997).

A system is in DC modelled within a conventional dynamical systems framework with states that are functions of time, the non-negative reals. A Boolean state is observed through its duration in a bounded interval, i.e. the integral of an indicator function. A property for a single interval is specified by an arithmetic relation among durations and real numbers. Properties for sequences of intervals are expressed by 
the associative binary "chop" operator of the Interval Temporal Logic (Moszkowski 1985).

In a specification of requirements, the dynamical systems model is beneficial, because it focuses on relevant observable states. Furthermore, the interval logic has simple interpretations in timing diagrams for the states. The requirements will thus focus on sequencing of durational relations between observable states rather than on state transitions. A typical real-time property is bounded critical duration: in every interval of at most length $\delta$ the duration of a state $P$ should not be more than $\gamma$ time units. In DC this is expressed by the formula:

$$
\ell \leq \delta \Rightarrow \int P \leq \gamma
$$

However, when dealing with an implementation represented as a state machine such formulas do not lend themselves directly to structural induction proof methodologies. Also, at a design level, stronger assumptions may be made about timing. For example, a finite number of state changes may be assumed to occur simultaneously at a point of time, though the order in which they occur is still significant. Therefore, properties at a real-time point becomes significant when one introduces time into widely accepted transition systems models for reactive systems in general, e.g. (Keller 1976). While transition systems and the linear temporal logic (LTL), e.g. (Manna \& Pnueli 1981), have been used successfully for specification and verification of reactive systems, they are not expressive enough for systems with real-time constraints, typically bounded invariance and bounded responsiveness.

Real-time is introduced into transition systems either by associating lower and upper bounds on enabled transitions (e.g. (Lamport 1977)) or by introducing explicit clocks, e.g. (Abadi \& Lamport 1992). The first approach has led to extensions of LTL with bounded operators (Koymans, Vytopil \& de Roever 1983), and the second has used LTL with explicit clocks, e.g. (Abadi \& Lamport 1992). The relationship between the two approaches is investigated in (Henzinger, Manna \& Pnueli 1994).

An advantage of TTS frameworks is that they are quite close to implementations using digital hardware and thus directly reflect semantics of programming languages. Another advantage is that safety properties and time bounded properties can be easily checked by structural induction over the transitions. However, this framework is inherently operational and less abstract than a dynamical systems based framework. For instance, properties that relate states across several transitions have to be expressed using auxiliary state variables (Lamport 1993). Thus it seems well justified to link the two formalisms such that one may use DC for high level specification and reasoning and TTS for implementation and refinement.

In a previous work (Sørensen, Hansen \& Løvengreen 1994), a link is demonstrated through definition of a combination of the logics of TLT and Duration Calculus. That work investigated some of the techniques presented here. However, the current work differs by focusing on the proof system and proof techniques, which turns out to be the difficult and important part when combining theories.

After this introduction, Section 2 gives an overview of DC and its application to high level specification. Section 3 introduces TTS and a model of their executions called trajectories, which allows us to define the satisfaction of a DC formula by a 
TTS in Section 4. In Section 5, we provide the rules for proving duration properties of a TTS. In Section 6, the Gas Burner example illustrates the approach and allow comparisons between this mixed approach and DC-only (Ravn et al. 1993) or TTSonly (Lamport 1993) methods.

\section{REQUIREMENT AND DESIGN SPECIFICATION IN DC}

A dynamic system is traditionally modelled by its state functions which are evaluations (or interpretations)of the system state variables $\Pi$ over the time domain, each of them has a signature $\mathcal{V}(x):$ Time $\longmapsto V$, where $V$ is the value domain of $x \in \Pi$.

The Time domain must be totally ordered, and any interval $\left[t_{1}, t_{2}\right]$ between two time points is measurable by a non-negative real number called the length of the interval. The usual interpretation is the set of non-negative real numbers $\mathbf{R} \geq 0$, but we extend it to $\mathbf{R}^{\geq 0} \times \mathbf{N}$ later in this paper.

A state function $\mathcal{V}(x)$ must be sectionally continuous and locally bounded. Sectional continuity means that in a bounded interval there is a finite number of discontinuities. For discrete state variables, which are the only variables that we use in this paper, a state function is a step function.

A state assertion $P$ is a first order predicate over the state variables $\Pi$ and interpreted a Boolean valued function $\mathcal{V}(P):$ Time $\longmapsto\{0,1\} ; \mathcal{V}(P)(t)=1$ if the evaluations of the variables in $P$ at $t$ make $P$ hold, and $\mathcal{V}(P)(t)=0$ otherwise. The sectional continuity implies that $\mathcal{V}(P)$ is a step function.

The elementary observation of a state assertion $P$ is its duration, written $\int P$. It denotes for a given time interval $\left[t_{1}, t_{2}\right]$, the integral $\int_{t_{1}}^{t_{2}} \mathcal{V}(P)(t) d t$. In particular, the largest duration corresponding to the length of the real-time interval is $\int 1$, which we abbreviate as $\ell$. The duration is a real number and a measure. It satisfies all the properties of a measure.

Elementary formulas are arithmetic relations in durations and real valued rigid variables and constants. An example is that $P$ holds throughout an interval: $\int P=\ell$.

The propositional connectives and quantification over rigid variables are used to generate composite formulas. An example is that $P$ holds on a proper (non-point) interval:

$$
\lceil P\rceil \stackrel{\text { def }}{=}\left(\int P=\ell\right) \wedge(\ell>0)
$$

Analogously, we introduce the abbreviation for a 'point':

$$
\lceil\rceil \stackrel{\text { def }}{=} \ell=0
$$

Properties of subintervals are specified by the binary 'chop'-operator, denoted by '; '. A formula $D_{1} ; D_{2}$ holds on an interval $\left[t_{1}, t_{2}\right]$ just when there exists an intermediate $t$ such that the formula $D_{1}$ holds on $\left[t_{1}, t\right]$ and the formula $D_{2}$ holds on $\left[t, t_{2}\right]$.

The chop operator and durations are linked by the key property of summation over subintervals: for any real numbers $\gamma, \delta \geq 0$

$$
\left(\int P=\gamma+\delta\right) \Leftrightarrow\left(\left(\int P=\gamma\right) ;\left(\int P=\delta\right)\right)
$$


For more details about the semantics, axioms and rules of DC, we refer the reader to (Hansen \& Zhou 1997).

\subsection{Specification of the Gas Burner}

We use the Gas Burner example in (Ravn et al. 1993) to show the simplicity in writing requirement and design specifications in DC. This case study formulate the safety requirement of a gas burner in terms of a variable Leak denoting an undesirable but unavoidable state where unlit gas escapes from the nozzle. Safety requires that 'gas must never leak for more than 4 seconds in any period of at most 30 seconds':

$$
R e q \stackrel{\text { def }}{=} \ell \leq 30 \Rightarrow \int \text { Leak } \leq 4
$$

To meet the requirement $R e q$, we make two design decisions:

$$
\begin{aligned}
& D e s_{1} \stackrel{\text { def }}{=}\lceil\text { Leak }\rceil \Rightarrow \ell \leq 4 \\
& D e s_{2} \stackrel{\text { def }}{=}\lceil\text { Leak }\rceil ;\lceil\neg L e a k\rceil ;\lceil L e a k\rceil \Rightarrow \ell \geq 26
\end{aligned}
$$

$D e s_{1}$ is a bounded response property: it says that any occurrence of Leak must be stopped within 4 seconds. The other formula $D s_{2}$ is a bounded invariance: it says that once a Leak is stopped, this persists for at least 26 seconds.

The correctness of the design, i.e. implication of the validity of $R e q$ by that of Des $\stackrel{\text { def }}{=} D e s_{1} \wedge D e s_{2}$, is shown in (Zhou et al. 1991) and for a more complex design in (Ravn et al. 1993).

The formula Des may be interpreted as a real-time automaton or a real-time program, which is refined further in a detailed design. However, this is in general a 'big' formula and a direct proof of refinement in DC may be tedious, because many of the steps deals with encoding of the behaviour of the automaton. We propose to deal with these steps by adopting the inductive proof techniques of the TTS framework.

\section{TRANSITION SYSTEMS}

This section starts with an overview of transition systems, as given in (Manna \& Pnueli 1992). As in (Henzinger et al. 1994) a transition system is then extended to a timed version by imposing timing constraints on its transitions. We deviate slightly from these sources when we define the behaviour of a TTS in a trajectory model which is convenient to use as a semantic model for DC in Section 4.

\subsection{Transition systems}

A transition system $S=\langle\Pi, \Sigma, \Theta, \mathcal{T}\rangle$ consists of four components:

1. II is a finite set of state variables. 
2. $\Sigma$ is a set of states. A state $s$ in $\Sigma$ assigns to every variable $u \in \Pi$ a value $s[u]$.

3. The initial condition $\Theta$ is a state assertion that defines a subset of $\Sigma$ called the initial states.

4. A finite set $\mathcal{T}$ of transitions. Every transition $\tau$ in $\mathcal{T}$ is a binary relation on $\Sigma$; it defines for every state $s$, a (possibly empty) set of $\tau$-successors $\tau(s)$. We use $e n(\tau)$ to denote the enabling condition of a transition $\tau$, and transition $\tau$ is enabled on a state $s$ just when $\tau(s) \neq \emptyset$. When a $\tau$ holds between states $s$ and $s^{\prime}$ this is often written $s \stackrel{\tau}{\rightarrow} s^{\prime}$.

A computation (execution, run) of a transition system $S$ is an infinite state sequence $\sigma=\sigma_{0}, \sigma_{1}, \cdots$ which satisfies the following two conditions:

Initiation: $\quad \sigma_{0}$ satisfies $\Theta$.

Consecution: For all $i \geq 0$, either $\sigma_{i}=\sigma_{i+1}$ (a stuttering step) or there is a transition $\tau$ in $\mathcal{T}$ such that $\sigma_{i} \stackrel{\tau}{\rightarrow} \sigma_{i+1}$ (a diligent step). In the later case, we say that a $\tau$ step is taken at position $i$ of $\sigma$.

Thus, a computation either contains infinitely many diligent steps, or it terminates with an infinite stuttering-only suffix. The set $\llbracket S \rrbracket$ of all computations of $S$ is stuttering closed: if an infinite state sequence $\sigma$ is a computation of the program, then so is any state sequence obtained from $\sigma$ by adding or deleting a finite number of stuttering steps. Stuttering closure is the key to relate system specifications (or models) (Abadi \& Lamport 1991) at different level of abstractions by refinement mappings.

As in Manna and Pnueli's Linear Temporal Logic (LTL) (Manna \& Pnueli 1992) or Lamport's Temporal Logic of Actions (TLA) (Lamport 1994), we represent transition $\tau$ of a system $S$ as first order Boolean-valued over the state variables $\Pi$ and their 'primed versions' $\Pi^{\prime}$, which is interpreted over pairs of states:

$$
\left\langle s, s^{\prime}\right\rangle \vDash \tau\left(\Pi, \Pi^{\prime}\right) \text { iff } \tau\left(s[\Pi] / \Pi, s^{\prime}[\Pi] / \Pi^{\prime}\right) \text { holds }
$$

\subsection{Timed transition systems}

We incorporate time in the transition systems models by assuming that all transitions happen "instantaneously", while timing constraints require transitions to be performed neither too early nor too late. Each transition $\tau$ is given a lower time bound $l_{\tau}$, and an upper time bound $u_{\tau}$. The lower time bound is a value from $\mathbf{R} \geq 0$, which we take to be the set of non-negative real numbers, and the upper time bound is either a value from $\mathbf{R}^{\geq 0}$ or the special symbol $\infty$, which denotes the absence of an upper bound. Any real number in $\mathbf{R}^{\geq 0}$ is assumed to be less than $\infty$, and the lower bound is assumed not to exceed the upper bound for any transition.

A timed transition system $T S=\langle\Pi, \Sigma, \Theta, \mathcal{T}, l, u\rangle$ consists of an underlying transition system $S=\langle\Pi, \Sigma, \Theta, \mathcal{T}\rangle$ and two functions $l$ and $u$ defining the transition time interval for each transition. 


\subsection{Trajectories}

Instead of defining a computations as a timed state sequence (Henzinger et al. 1994), we introduce the notion of a trajectory in order to interpret DC formulas over behaviours of a TTS. A trajectory over a set $\Pi$ of state variables is a state function: $\rho:$ Time $\Sigma$, where $\Sigma$ is the set of states.

The time domain is Time $\stackrel{\text { def }}{=} \mathbf{R}^{\geq 0} \times \mathbf{N}$ in this framework. We refer to a pair $\langle t, n\rangle \in$ Time as a position, and $\rho(t, n)$ as the state at position $\langle t, n\rangle$ in $\rho$. Positions in Time are ordered by the lexicographic ordering: $\left\langle t_{1}, n_{1}\right\rangle \prec\left\langle t_{2}, n_{2}\right\rangle$ iff $t_{1}<t_{2}$ or $\left(t_{1}=t_{2}\right) \wedge\left(n_{1}<n_{2}\right)$. We write $\left\langle t_{1}, n_{1}\right\rangle \preceq\left\langle t_{2}, n_{2}\right\rangle$ if $\left\langle t_{1}, n_{1}\right\rangle \prec\left\langle t_{2}, n_{2}\right\rangle$ or $\left\langle t_{1}, n_{1}\right\rangle=\left\langle t_{2}, n_{2}\right\rangle$. For two positions $p_{1}$ and $p_{2}$ such that $p_{1} \preceq p_{2}$, the super-dense time interval $\left[p_{1}, p_{2}\right]$ between $p_{1}$ and $p_{2}$ is the set of positions $\left\{p: p_{1} \preceq p \preceq p_{2}\right\}$. We also define the length $d_{1}\left(\left\langle t_{1}, n_{1}\right\rangle,\left\langle t_{2}, n_{2}\right\rangle\right)$ of an interval $\left[\left\langle t_{1}, n_{1}\right\rangle,\left\langle t_{2}, n_{2}\right\rangle\right]$ as $t_{2}-t_{1}$.

In order to make a trajectory $\rho$ isomorphic to a timed state sequence in (Henzinger et al. 1994), we impose onto it the sectional continuity (or finite variability) condition: For any interval $[\langle a, m\rangle,\langle b, n\rangle]$, there exits a finite number of positions $\left\langle t_{1}, n_{1}\right\rangle, \cdots,\left\langle t_{k}, n_{k}\right\rangle$ such that

1. $a=t_{1}<t_{2}<, \cdots,<t_{k}=b$, and

2. $\rho$ is constant on each interval $\left[\left\langle t_{i}, n_{i}\right\rangle,\left\langle t_{i+1}, 0\right\rangle\right]$.

Define the projection $\rho(t), t \in \mathbf{R}^{\geq 0}$, as $\rho(t, 0)$. Then the above two conditions ensure that: (a) a state change can only occur between two consecutive positions $\langle t, n\rangle$ and $\langle t, n+1\rangle$, for some $t \in \mathbf{R}^{\geq 0}$ and some $n \in \mathbf{N}$; (b) the state sequence at a time point $t \in \mathbf{R}^{\geq 0}$ terminates with a constant state, denoted as $\rho(t, \infty)$; and (c) the first state $\rho(t)$ at a time point $t$ equals the constant state of the preceding evolution: $\rho(t)=\rho\left(t^{-}\right)$; and the final state is the value of the succeeding evolution: $\rho(t, \infty)=\rho\left(t^{+}\right)$.

If $\rho(t, n) \stackrel{\tau}{\rightarrow} \rho(t, n+1)$, we say transition $\tau$ is taken at position $\langle t, n\rangle$ in $\rho$, and $\tau$ is taken at time point $t$ in $\rho$ if there exists a $n$ such that $\tau$ is taken at $\langle t, n\rangle$ in $\rho$.

A trajectory $\rho:$ Time $\longmapsto \Sigma$ is a trajectory of a timed transition system, TS = $\langle\Pi, \Sigma, \theta, \mathcal{T}, l, u\rangle$, if it satisfies the following conditions.

Initiation: $\quad \rho(0,0)$ satisfies $\Theta$.

Consecution: For any position $\langle t, n\rangle$, either $\rho(t, n)=\rho(t, n+1)$ (a stuttering step) or there is a $\tau \in \mathcal{T}$ such that $\rho(t, n) \stackrel{\tau}{\rightarrow} \rho(t, n+1)$ (a diligent step).

Lower bound: if $\tau$ is taken at position $p$, there must exist a $p_{1} \preceq p$ such that $d_{1}\left(p, p_{1}\right) \geq l_{\tau}$, and for any $p_{2}$ such that $p_{1} \preceq p_{2} \prec p, \tau$ is not taken.

Upper bound: if $\tau$ is enabled at position $p$, there exists $p_{1} \succeq p$ such that $d_{1}\left(p_{1}, p\right) \leq$ $u_{\tau}$ and $\tau$ is taken or is disabled at $p_{1}$.

A trajectory $\rho$ of $T S$ that satisfies the first two conditions is a trajectory of its un- 
derlying transition system. It is easy to see that the set $\llbracket T S \rrbracket^{t r a j}$ of all trajectories of a system $T S$ is isomorphic to the set of timed state sequences of $T S$ defined in (Henzinger et al. 1994).

Intuitively, the design decisions of Des for the Gas Burner system are implemented by a two-state and two-transition TS, denoted as $G B_{1}$, which is formally described below in terms of its initial condition and transitions with their time bounds:

$$
\begin{aligned}
& G B_{1}=\left\langle\Theta_{1}:\right. \text { true } \\
& \tau_{1}: \text { Leak } \wedge \neg \text { Leak' }, \quad l_{\tau_{1}}=0, \quad u_{\tau_{1}}=4 \\
& \tau_{2}: \neg \text { Leak } \wedge \text { Leak', } l_{\tau_{2}}=26, \quad u_{\tau_{2}}=\infty \\
& >
\end{aligned}
$$

The problem is, within a DC framework, to formally prove that $G B_{1}$ satisfies $D e s$ and then how to refine it.

\section{SATISFACTION OF A DC FORMULA BY A TTS}

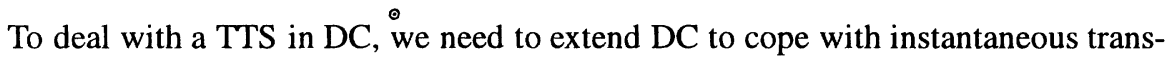
itions, and thus sequences of point properties. This section defines DC in terms of trajectories of transition systems and define the satisfaction relation of a duration formula by a TTS. This semantic definition preserves all the validity of the original DC axioms and rules.

\subsection{Syntax}

We keep the concept of a state assertion $P$, its duration $\int P$, rigid variables $v$, and define as usual an atomic duration formula $F$ as any arithmetic relation over real valued constants, durations and rigid variables.

To describe state changes in a point interval, a transition $\tau$, as well as an integer arithmetic relation over the step counter $\hbar$ and rigid variables, are also atomic formulas.

In addition to the binary "chop" modality, denoted by '; ', for specifying properties of subintervals, We introduce the unary modality $\triangleright$ which allows us to either prefixing or extending an interval.

A DC formula $D$ is generated from the atomic formals by the Boolean connectives, the binary chop modality, the unary modality $\triangleright$, and quantification over rigid variables.

\subsection{Semantics}

A formula of this extended logic is to be interpreted over a trajectory $\rho$ and a superdense interval $\left[p_{1}, p_{2}\right]$ between two positions $p_{1}=\left\langle t_{1}, n_{1}\right\rangle$ and $p_{2}=\left\langle t_{2}, n_{2}\right\rangle$. We 
define two measures of such an interval by the two-valued function

$$
d\left(p_{1}, p_{2}\right) \stackrel{\text { def }}{=} \begin{cases}\left(t_{2}-t_{1}, n_{2}\right) & \text { if } t_{1}<t_{2} \\ \left(0, n_{2}-n_{1}\right) & \text { if } t_{1}=t_{2} \wedge n_{1} \leq n_{2}\end{cases}
$$

We shall use $d_{1}\left(p_{1}, p_{2}\right)$ and $d_{2}\left(p_{1}, p_{2}\right)$ to denote the first (the length of the interval) and second (the steps of the interval) values of $d\left(p_{1}, p_{2}\right)$ respectively.

For a trajectory $\rho$ over a state space $\Sigma$, an observation over an interval $\left[p_{1}, p_{2}\right]$ consists of the states $\left\{\rho(t, n): p_{1} \preceq\langle t, n\rangle \preceq p_{2}\right\}$. In particular, at any time point $t$, every observation over an interval $\left[\left\langle t, n_{1}\right\rangle,\left\langle t, n_{2}\right\rangle\right]$ (which is a point interval) is a finite non-empty state sequence $\rho\left(t, n_{1}\right), \cdots, \rho\left(t, n_{2}\right)$. The observation of $\rho$ over $[\langle t, n\rangle,\langle t, n\rangle]$ is the single state $\rho(t, n)$, i.e. a state sequence of 'length 0 ' is a single state.

\section{Durations of states}

The duration $\int P$ for a state assertion $P$ over a super-dense interval $\left[\left\langle t_{1}, n_{1}\right\rangle,\left\langle t_{2}, n_{2}\right\rangle\right]$ is still used to define the accumulated time when $P$ holds within the super-dense interval:

$$
\int_{t_{1}}^{t_{2}} P(\rho(t, 0)) d t
$$

Thus, a duration ignores all the discontinuous positions, and its existence is guaranteed by the finite variability of the trajectory*. Under these definitions, all the conventional DC axioms in (Zhou et al. 1991) remain valid.

\section{State properties and transitions}

A state property $P$ which is a first order predicate assertion (no quantification is allowed over state variables) holds for a trajectory $\rho$ over an observation if it holds at the first position of the observation:

$$
\rho,\left[\left\langle t_{1}, n_{1}\right\rangle,\left\langle t_{2}, n_{2}\right\rangle\right] \models P \quad \text { iff } \quad \rho\left(t_{1}, n_{1}\right) \models P
$$

A transition $\tau$ is treated as an interval formula as for the case of a state predicate:

$$
\rho,\left[\left\langle t_{1}, n_{1}\right\rangle,\left\langle t_{2}, n_{2}\right\rangle\right] \models \tau \quad \text { iff } \quad\left\langle\rho\left(t_{1}, n_{1}\right), \rho\left(t_{1}, n_{1}+1\right)\right\rangle \vDash \tau
$$

Obviously, a state predicate is a special transition which does not refer to primed variables, and all axioms in the first order predicate logic remain valid for reasoning about state properties and transitions.

The composite formula \lceil\rceil$\wedge(\hbar=0) \wedge \tau$ specifies the point observations containing a single state in which $\tau$ is enabled; and the formula \lceil\rceil$\wedge(\hbar>0) \wedge \tau$ defines the point observations consisting of a sequence of more than one state such that the first two of them is a $\tau$-step.

The Hoare Triple $\{P\} \tau\{Q\}$ for any state assertions $P$ and $Q$ and any state transition $\tau$ will be used in structural induction reasoning, and it is defined here as the

*The logic remains the same if $\int P$ is defined as $\int_{t_{1}}^{t_{2}} \forall n \in \mathbf{N} \bullet P(\rho(t, n)) d t$ or as $\int_{t_{1}}^{t_{2}} \exists n \in \mathbf{N} \bullet$ $P(\rho(t, n)) d t$. 
state transition

$$
\{P\} \tau\{Q\} \stackrel{\text { def }}{=} P \wedge \tau \Rightarrow Q^{\prime}
$$

where $Q^{\prime}$ is obtained from $Q$ by replacing each state variable $x$ of $Q$ by its primed version $x^{\prime}$.

The length of a state sequence in an interval is given by the step counter $\hbar$ :

$$
\rho,\left[p_{1}, p_{2}\right] \models \hbar=n \quad \text { iff } \quad d_{2}\left(p_{1}, p_{2}\right)=n
$$

The bounded version of the temporal formula $\bigcirc D$ (i.e. the 'next' operator) can be defined in terms of \lceil\rceil$, \hbar$ and the chop operator as $(\lceil\rceil \wedge \hbar=1) ; D$.

\section{Modalities}

The 'chop' operator now chops a super-dense interval. A formula $D_{1} ; D_{2}$ holds on such an interval $\left[p_{1}, p_{2}\right]$ just when there exists an intermediate position $p$ such that the formula $D_{1}$ holds on $\left[p_{1}, p\right]$ and the formula $D_{2}$ holds on $\left[p, p_{2}\right]$ :

$$
\begin{aligned}
& \rho,\left[p_{1}, p_{2}\right] \models D_{1} ; D_{2} \text { iff } \\
& \exists p \bullet\left(p_{1} \preceq p \preceq p_{2}\right) \wedge\left(\rho,\left[p_{1}, p\right] \models D_{1}\right) \wedge\left(\rho,\left[p, p_{2}\right] \models D_{2}\right)
\end{aligned}
$$

A formula $\triangleright D$ holds on an interval $[b, e]$ there is an interval $[b, f]$ which is either a prefix or an extension of $[b, e]$ such that $D$ holds on $[b, f]$ :

$$
\rho,[b, e] \models \triangleright D \quad \text { iff } \quad \exists f \succeq b \bullet(\rho,[b, f] \models D)
$$

This operator distributes over disjunction and is monotone. It comes as a combination $\diamond_{l} \diamond_{r}$ of the two neighbourhood modalities $\diamond_{l}$ and $\diamond_{r}$ from (Zhou \& Hansen $1996 a$ ). In this paper, we are only concerned about future properties which can be treated sufficiently by the combined operator. Axioms and properties for the chop operator and the $\triangleright$ operator (treated as $\diamond_{l} \diamond_{r}$ ) given in (Zhou et al. 1991) and (Zhou \& Hansen 1996a) remain valid under the new interpretation.

With operator $\triangleright$, the conventional linear temporal formulas $\diamond D$ and $\square D$ can be defined as follows $\triangleright($ true ; $D)$ and $\neg \diamond \neg D$, respectively.

A trajectory $\rho$ satisfies a formula $D$ if for any $\langle r, n\rangle \in$ Time $\rho,[\langle 0,0\rangle,\langle r, n\rangle] \models$ $D$. Valid formulas are satisfied by any trajectory. A formula $D$ is validfor (or satisfied by) a TTS, TS, denoted by $T S \vDash D$, iff any trajectory $\rho \in \llbracket T S \rrbracket^{t r a j}$ satisfies $D$.

\subsection{Proof System}

We have just said that in the extended logic, the validity of the axioms and rules in the original DC in (Moszkowski 1985, Zhou et al. 1991, Zhou \& Hansen 1996a) for durations and the two modalities is preserved as well as the validity of the axioms and rules in first predicate logic is for state properties and transitions at a time point. For example, it is easy to see that as in original DC and ITL, 'chop' is associative, distributes over disjunction, and has false as zero. Furthermore, for any durational formula $D,\lceil\rceil$ is the unit of 'chop': $\rceil ; D \Leftrightarrow D ;\lceil\rceil \Leftrightarrow D$. However, we need 
axioms to link point properties with interval properties. The following axioms about temporal variable $\hbar$ play this role:

A1: $\exists n: \mathbf{N} \bullet(\hbar=n)$

A2: $(D \Leftrightarrow\lceil\rceil \wedge(\hbar=0) ; D) \wedge(D \Leftrightarrow D ;\lceil\rceil \wedge(\hbar=0)) \quad$ unit of chop

A3 : $\quad\lceil\rceil \wedge(\hbar=n>0) \Leftrightarrow(\lceil\rceil \wedge \hbar=1 ;\lceil\rceil \wedge(\hbar=n-1))$

\lceil\rceil$\wedge(\hbar=n>0) \Leftrightarrow(\lceil\rceil \wedge \hbar=n-1 ;\lceil\rceil \wedge(\hbar=1))$ chop a point

A4 : $\quad(\ell=r) \Rightarrow \exists n \bullet(\lceil\rceil \wedge(\hbar=n) ;(\ell=r))$

super-dense

A5 : $\neg(\lceil\rceil \wedge \hbar=0) \Rightarrow((\{P\} \tau\{Q\}) \Leftrightarrow(P \wedge \tau \Rightarrow \bigcirc Q)) \quad$ link positions

For a state predicate $P$ we use $P^{\bullet}$ to denote that $P$ holds anywhere in a interval:

$P^{\bullet} \stackrel{\text { def }}{=} \neg($ true $; \neg P ;$ true $)$

\section{LIFTING PROPERTIES OF TTS TO DURATION PROPERTIES}

The 'yardstick' properties of a timed transition system include bounded invariance properties, and bounded progress properties. These properties and the structural induction proof rules for them are given in (Henzinger et al. 1994) in timed linear temporal logic (TLTL). This section shows how these properties are incorporated into the extended DC framework.

A state property $P$ is an invariant of a transition system $S$ if $P$ holds at any position in any interval for all trajectories of $S$. Thus, an unbounded invariance property is of the form $\square P$, where $P$ is a state predicate. $P$ is an invariant of a transition system $S$ iff $P$ is implied by $\Theta$, and $P$ is preserved by all transitions in $\mathcal{T}$. Let $\{P\} \mathcal{T}\{Q\}$ hold iff $\mathcal{T}\{P\} \tau\{Q\}$ holds for each $\tau \in \mathcal{T}$. An induction rule for proving an invariant of $S$ is given in (Manna \& Pnueli 1981) as:

UB-INV

$$
\frac{\Theta \Rightarrow P, \quad\{P\} \mathcal{T}\{P\}}{\square P}
$$

which is still sound in the interval logical setting, i.e. any transition system satisfying the premises also satisfies the conclusion.

There are other induction rules for proving untimed properties which we leave out of this paper, because our goal is the time bounded properties. We are mainly concerned with bounded properties.

As in (Henzinger et al. 1994), we are interested in proving bounded-invariance and bounded-response properties, and thus restrict ourselves to the following bounded temporal formulas:

Primitive formulas: State formulas and state transitions are (linear) temporal formulas.

Boolean connectives: Every Boolean combination of temporal formulas is a temporal formula.

Bounded-eventually formulas: If $\phi$ is temporal formula and $u \in \mathbf{R}^{\geq 0}$, then so is $\diamond_{\leq u} \phi$; it is true over a trajectory $\rho$ with an initial position $\left\langle t_{0}, n_{0}\right\rangle$ iff there is a position $p=\langle t, n\rangle$ such that $t \leq t_{0}+u$ and $\phi$ holds for the suffix of $\rho$ starting with 
$p$. We thus define

$$
\begin{aligned}
& \diamond_{\leq u} \phi \stackrel{\text { def }}{=}(\ell<u) \vee(\ell \leq u ; \phi) \quad \text { or equivalently } \\
& \diamond_{\leq u} \phi \stackrel{\text { def }}{=} \triangleright(\ell \leq u ; \phi)
\end{aligned}
$$

Bounded-unless formulas: If $P$ is a state properties, $\phi$ is a temporal formula, and $l \in \mathbf{R} \geq 0$, then $P \mathcal{U}_{\geq l} \phi$ is a temporal formula; it is true over a trajectory $\rho$ iff $P$ holds at the initial position $\left\langle t_{0}, n_{0}\right\rangle$ of $\rho$ and either $P$ holds for all positions in $\rho$, or there there is some position $p$ such that $t \geq t_{0}+l$, and $\phi$ holds for the suffix starting with $p$, and $P$ holds at every position $q \prec p$. This formula can thus be defined as

$$
P \mathcal{U}_{\geq l} \phi \stackrel{\text { def }}{=}\left(P^{\bullet} \vee\left(P^{\bullet} \wedge(\ell \geq l) ; \bigcirc \phi\right)\right)
$$

We use the convention that the letters $P, Q, R$, as well as $\varphi$, denote state formulas; $\tau$ and its indexed versions denote state transitions; $\phi, \psi$ and $\chi$ stand for temporal formulas; and $D, F$ and their indexed versions, denote general formulas in the extended logic. We also denote $P \mathcal{U}_{\geq l}$ true by $\square_{<l} P$ when $l>0$, and define $\square_{<0} P$ to be true.

\subsection{Induction rules for bounded properties}

We start with bounded invariance properties. A bounded invariance property asserts that when some state occurs a condition holds continuously for a certain amount of time. Such a property is specified by a temporal formula of the form $P \Rightarrow \square{ }_{l l} Q$. Therefore, to prove a bounded invariance property, we often have to prove a bounded unless property. There are five main rules for bounded unless in (Henzinger et al. 1994) given in Table 1, which are still sound in our setting.

\begin{tabular}{ll}
\hline $\mathcal{U}$-MON & $\frac{P \Rightarrow P_{1}, \phi \Rightarrow \phi_{1}, l_{1} \leq l}{\left(P \mathcal{U}_{>l} \phi\right) \Rightarrow\left(P_{1} \mathcal{U}_{\left.\geq l_{1} \phi_{1}\right)}\right.}$ \\
$\mathcal{U}$-TRAN & $\frac{\varphi \Rightarrow P \mathcal{U}_{\geq l_{1}} \chi, \chi \Rightarrow \mathcal{U}_{\geq l_{2}} \psi}{\varphi \Rightarrow(P \vee Q) \mathcal{U}_{\geq l_{1}+l_{2}} \psi}$ \\
$\mathcal{U}$-SS & $\frac{P \Rightarrow \neg e n(\tau), P \Rightarrow \varphi, \varphi \Rightarrow Q,\{\varphi\} \mathcal{T}-\tau\{\varphi\},\{\varphi \wedge e n(\tau)\} \tau\{R\}}{P \Rightarrow Q \mathcal{U}_{\geq l_{\tau}} R}$ \\
$\mathcal{U}$-CSS & $\frac{P \Rightarrow \neg e n(\tau),\{Q\} \mathcal{T}-\tau\{\neg R\}^{-}}{P \mathcal{U}_{\geq l} Q \mathcal{U}(R \wedge \phi) \Rightarrow P \mathcal{U}_{\geq l} Q \mathcal{U}_{\geq l_{\tau}}(R \wedge \phi)}$ \\
$\mathcal{U}$-COLL & $\left(P \mathcal{U}_{\geq l_{1}} Q \mathcal{U}_{\geq l_{2}} \phi\right) \Rightarrow\left((P \vee Q) \mathcal{U}_{\geq l_{1}+l_{2}} \phi\right)$ \\
\hline
\end{tabular}

Table 1 Rules for bounded unless

A bounded progress property asserts that once $P$ occurs, $Q$ must occur within a certain amount of time, and thus is specified in the form $P \Rightarrow \diamond_{\leq u} P$. Again, the rules for bounded progress properties in (Henzinger et al. 1994) can be brought into our framework, which are given in Table 2. 


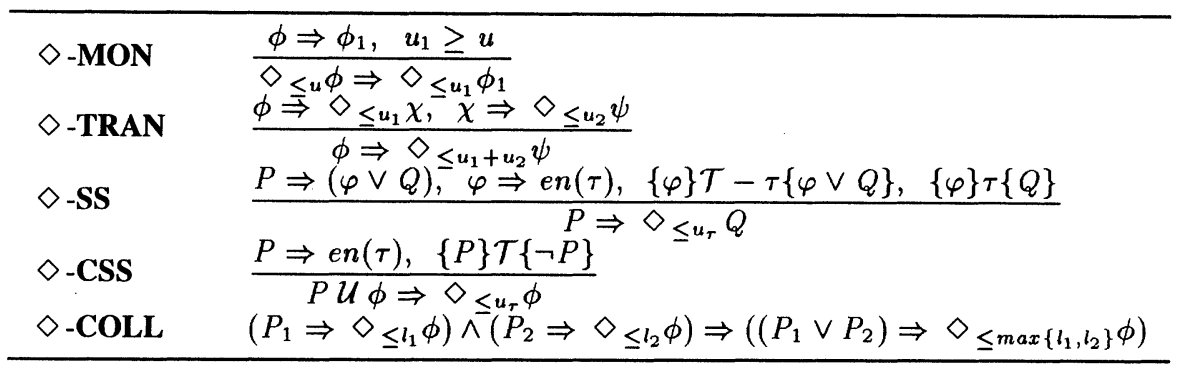

Table 2 Rules for bounded progress

\subsection{Linking rules}

Now we are ready to provide the rules which link temporal properties to the DCinvariance properties, DC-bounded response properties, and the DC-minimal separation properties. The rules are presented in the form such that the premises of a rule are temporal formulas (including first order predicates) and the conclusion is an formula in DC. This indicates that an establishment of a DC property can be achieved from some TLTL properties which can be proven by the structural induction rules.

The DC-invariance property \lceil\rceil$\vee\lceil P\rceil$ can also be derived from the temporal invariance property $\square P$ :

\section{LINK-1}

$$
\frac{\square P}{\lceil\rceil \vee\lceil P\rceil}
$$

A DC-bounded response property is of the form $\neg(\lceil P\rceil ; \quad(\lceil\neg Q\rceil \wedge \ell>u))$, asserting that $P$ must be followed within $u$ time units by an occurrence of $Q$, and can be proved by the following linking rule:

LINK-2 $P \Rightarrow \diamond_{\leq u} Q$

$$
\frac{\neg Q \Rightarrow \neg Q \mathcal{U} Q \mathcal{U}_{\geq l} \neg Q, \quad \text { for some } l>0}{\neg(\lceil P\rceil ;(\lceil\neg Q\rceil \wedge \ell>u))}
$$

A minimal separation property is described as a DC-formula of the form

$$
\neg((\lceil P\rceil ;\lceil Q\rceil) \wedge(\ell<l) ; D ; \text { true })
$$

which asserts that $D$ cannot hold until $Q$ has been stable for at least $l$ time units since the change from a $P$-state to a $Q$-state took place. Using bounded unless properties, we can prove a minimal separation property by the following link:

LINK-3

$$
\begin{aligned}
& Q \Rightarrow \neg P, \quad \phi \Rightarrow \neg Q \\
& \psi \Rightarrow P \mathcal{U}_{\geq l_{1}} Q \mathcal{U} \phi \\
& P \mathcal{U}_{\geq l_{1}} Q \overline{\mathcal{U}} \phi \Rightarrow P \mathcal{U}_{\geq l_{1}} Q \mathcal{U} \geq_{l_{2}} \phi \\
& (\psi ; \text { true }) \Rightarrow \neg\left(([P\rceil ;\lceil Q\rceil) \wedge\left(\ell<l_{1}+l_{2}\right) ; \phi ; \text { true }\right)
\end{aligned}
$$

This rule is inductive in the sense that $\phi$ and $\psi$ are temporal formulas which may be linked further to DC-formulas. The first two premises $Q \Rightarrow \neg P$ and $R \Rightarrow \neg Q$ 
ensure the pattern $(\lceil P\rceil ;\lceil Q\rceil ; \phi)$ does not collapse, the premise in the second line says that a $\psi$-state sequence pattern can only proceed with $P$ lasting for at least $l_{1}$ time units followed by a $Q$-state and then a $\phi$ pattern, the premise in the third line then ensures that $Q$ lasts for at least $l_{2}$ time units.

The last two premises in the rule may be combined into one. However, the one in the second line have to be established which then can be used to establish the last one by rule $\mathcal{U}$-CSS. The special case when $l_{1}=0$ and $\psi$ is $P$ is often used. In this case the rule becomes

$$
\begin{array}{ll}
\text { LINK-3' }^{\prime} & Q \Rightarrow \neg P \quad R \Rightarrow \neg Q \\
& P \Rightarrow P \mathcal{U} Q \mathcal{U} R \\
& P \mathcal{U} Q \mathcal{U} R \Rightarrow P \mathcal{U} Q \mathcal{U}{ }_{\geq l} R \\
& \frac{(\lceil P\rceil ;\lceil Q\rceil ;\lceil R\rceil) \Rightarrow(\ell \geq l)}{}
\end{array}
$$

\section{IMPLEMENTATION AND REFINEMENT OF THE GAS BURNER}

This section shows how the linked methods are used in the development of the Gas Burner. We first show that the timed transition system $G B_{1}$ given in Section 3.2 satisfies the specification Des in Section 2.1. Then we show how $G B_{1}$ is refined into an implementation that may have only ignition failure. Finally, we present an implementation of Des which tolerates both ignition and flame failures.

Theorem 1 The timed transition system $G B_{1}$ given in Section 3.2, satisfies Des 1 and $\mathrm{Des}_{2}$.

This theorem follows from the following lemma:

Lemma 1 The time transition system $G B_{1}$ satisfies the following TLTL properties:

$$
\begin{array}{ll}
\left(T_{1}\right): & \text { Leak } \Rightarrow \diamond \leq 4 \neg \text { Leak } \\
\left(T_{2}\right): & \text { Leak } \Rightarrow \text { Leak } \mathcal{U} \neg \text { Leak } \mathcal{U} \text { Leak } \\
\left(T_{3}\right): & \text { Leak } \Rightarrow \text { Leak } \mathcal{U} \neg \text { Leak } \mathcal{U} \geq{ }_{26} \text { Leak }
\end{array}
$$

Proof: $\quad\left(T_{1}\right)$ is proven by applying $\diamond$-SS to $\tau_{1} \stackrel{\text { def }}{=}$ Leak $\wedge \neg L e a k^{\prime}$ and $u_{\tau_{1}}=4$, with the following premises:

$$
\begin{aligned}
& \text { Leak } \Rightarrow e n\left(\tau_{1}\right) \quad \text { as } e n\left(\tau_{1}\right)=\text { Leak } \\
& \{L e a k\} \tau_{2}\{L e a k\} \quad \text { as } L e a k \wedge \neg \text { Leak } \wedge \text { Leak' } \Rightarrow \text { Leak' } \\
& \{L e a k\} \tau_{1}\{\neg \text { Leak }\} \quad \text { as Leak } \wedge \text { Leak } \wedge \neg \text { Leak' } \Rightarrow \neg L e a k^{\prime}
\end{aligned}
$$

$\left(T_{2}\right)$ is obviously valid in the untimed LTL proof system. From $\left(T_{2}\right)$, we then have $\left(T_{3}\right)$ by applying $\mathcal{U}$-CSS for $\tau_{2}$ with $l_{\tau_{2}}=26 . \varnothing$

Proof of Theorem 1: Des $s_{1}$ is deduced by LINK-2 with $\left(T_{1}\right)$ and $\left(T_{3}\right)$ as the premises. $D e s_{2}$ follows from LINK-3' by letting $P, Q$, and $R$ in the rule be $L e a k, \neg L e a k$ and 
Leak, respectively, to establish the first two premises of the rule; while $\left(T_{2}\right)$ and $\left(T_{3}\right)$ are the last two premises.

\section{An implementation without flame failure}

After the initial implementation, $G B_{1}$ can be refined in the traditional TTS framework. For example, the transition system $G B_{2}$ in Figure 1 is a refinement of $G B_{1}$.

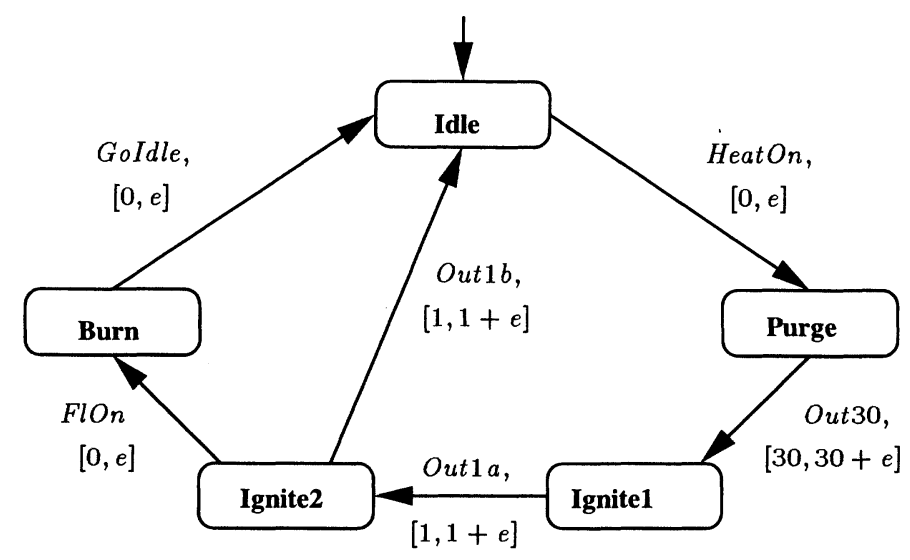

Figure 1 A refinement of $G B_{1}$

$G B_{2}$ has the following phases:

Idle: Await heat request, no gas and ignition. It enters Purge within $e$ time units on heat request. The constant parameter $e$ in this example is the system wide upper bound for reacting*.

Purge: Pauses for 30 seconds and then enters Ignite1 within $e$ time units.

Ignite1: Turns on ignition and gas supply and enters after one second the Ignite2 phase within $e$.

Ignite2: Monitors the flame and enters Burn if it is sensed within 1 second, otherwise returns to Idle while turning the gas off within $e$.

Burn: Ignition is switched off but gas is still on. The Burn phase is stable until heat request goes off. Gas is then turned off and Idle is entered within $e$.

We use a simple error recovery: return to Idle from Ignite2. We assume no flame failure in the Burn phase. Therefore, in this implementation, Leak can only occur in the Ignite1 and Ignite2 phases.

*In (Ravn et al. 1993, Lamport 1993), a lower bound is also given. It is not needed for proving the correctness of this design. 
With the convention that the value of a variables $x$ is changed by a transition $\tau$ only if $\tau$ names $x^{\prime}$, the formal definition of this refined transition systems is as follows.

$$
\begin{aligned}
& G B_{2}=\langle\Pi \quad \stackrel{\text { def }}{=} \quad\{\text { gas, ignition, sensor, phase }\} \\
& \Theta \quad \stackrel{\text { def }}{=} \text { (gas, ignition, sensor, phase })=\text { (off, off, off, Idle) } \\
& \text { HeatOn } \stackrel{\text { def }}{=} \text { HeatReq } \wedge(\text { phase }=\text { Idle }) \wedge\left(\text { phase }^{\prime}=\text { Purge }\right) \\
& \text { Out30 } \stackrel{\text { def }}{=} \quad(\text { phase }=\text { Purge }) \wedge \\
& \left(\text { phase }^{\prime}, \text { gas }^{\prime}, \text { ignition }^{\prime}\right)=(\text { Ignite1 }, \text { on }, \text { on }) \\
& \text { Out } 1 a \stackrel{\text { def }}{=}(\text { phase }=\text { Ignite1 }) \wedge\left(\text { phase }^{\prime}=\text { Ignite2 }\right) \wedge \\
& \text { (sensor' }=\text { on) } \\
& \text { Out } 1 b \stackrel{\text { def }}{=} \quad(\text { phase }=\text { Ignite } 2) \wedge\left(\text { phase } e^{\prime}=\text { Idle }\right) \wedge \\
& \text { (ignition } \left.{ }^{\prime}=\text { off }\right) \wedge\left(\text { gas }^{\prime}=\text { off }\right) \\
& \text { FlOn } \stackrel{\text { def }}{=}(\text { phase }=\text { Ignite } 2) \wedge \text { Flame } \wedge\left(\text { phase }^{\prime}=\text { Burn }\right) \wedge \\
& \text { (ignition' }=\text { off) } \\
& \text { GoIdle } \stackrel{\text { def }}{=}(\text { phase }=\text { Burn }) \wedge \neg \text { HeatReq } \wedge\left(\text { phase }{ }^{\prime}=\text { Idle }\right) \wedge \\
& \left(\text { gas }^{\prime}=\text { off }\right) \wedge\left(\text { sensor }{ }^{\prime}=\text { off }\right)
\end{aligned}
$$

\begin{tabular}{|c|c|c|}
\hline & Property of $G B_{2}$ & Property of $G B_{1}$ \\
\hline$\left(T_{11}\right)$ & $L_{e a k_{1}} \Rightarrow \diamond_{\leq 1+e} \neg$ Leak $k_{1}$ & $T_{1}$ of $L e a k$ \\
\hline$\left(T_{12}\right)$ & $\operatorname{Leak}_{1} \Rightarrow \operatorname{Le}_{\bar{a}} k_{1} \mathcal{U} \neg$ Leak $_{1} \mathcal{U}$ Leak $_{1}$ & $T_{2}$ of $L e a k$ \\
\hline & $\operatorname{Leak}_{1} \Rightarrow$ Leak $_{1} \mathcal{U} \neg$ Leak $\mathcal{H}_{1} \mathcal{U}_{\geq 31}$ Leak $_{1}$ & $T_{3}$ of $L e a k$ \\
\hline$\left(T_{21}\right.$ & Leak $_{2} \Rightarrow \diamond_{\leq 1+e} \neg$ Leak 2 & $T_{1}$ of $L e a k$ \\
\hline$\left(T_{22}\right.$ & Leak $_{2} \Rightarrow$ Leak $_{2} \mathcal{U} \neg$ Leak 2 U Leak & $T_{2}$ of Leak \\
\hline$T_{T}$ & Leak $_{2} \Rightarrow$ Leak $_{2} \mathcal{U} \neg$ Leak $k_{2} \mathcal{U} \geq{ }_{31} L_{e a k_{2}}$ & $T_{3}$ of Leak \\
\hline
\end{tabular}

The time bounds are given by Figure 1 .

Let $L e a k_{1}$ hold iff phase = Ignite1 holds and Leak 2 hold iff phase = Ignite2 holds. In the following, we present the properties of these two leak states of $G B_{2}$ which correspond to the properties of Leak and have same proof routines as those for $G B_{1}$.

Lemma 2 The timed transition system $G B_{2}$ satisfies the following TLTL properties provided $e \leq 1$ :

By the induction rules, Lemma 2 leads to the following corollary:

Corollary $1\left(G B_{2}\right.$ refines $\left.G B_{1}:\right)$ Let Leak $\stackrel{\text { def }}{=} L e a k_{1} \vee L e a k_{2}$ and $e \leq 1 . G B_{2}$ satisfies the properties $\left(T_{1}\right),\left(T_{2}\right)$ and $\left(T_{3}\right)$ in Lemma 1 .

Thus, we have the following theorem corresponding to Theorem 1 for $G B_{1}$. 
Theorem 2 Let Leak $\stackrel{\text { def }}{=} L_{e a k_{1}} \vee L e a k_{2}$ and $e \leq 1$. GB $B_{2}$ satisfies Des Ind Des $_{2}$, as well as Req, of the Gas Burner system.

Lemma 3 The timed transition system $G B_{2}$ has the following properties if $e \leq 1$ :

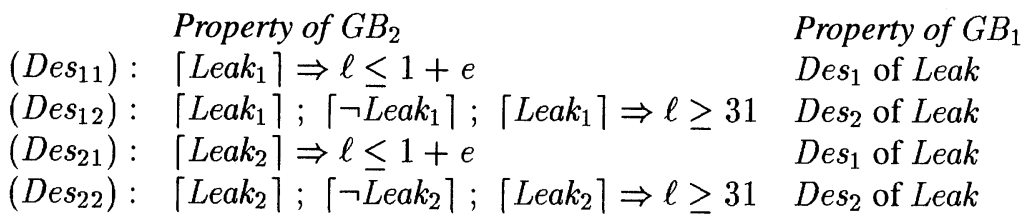

Using Lemma 2, the proofs of $D e s_{11}$ and $D e s_{21}$ are the same as the proof of $D e s_{1}$ of $G B_{1}$, while the proofs of $D e s_{12}$ and $D e s_{22}$ are the same as that of $D e s_{2}$ for $G B_{1}$, in Theorem 1.

Using the linking rules as in the proof of Theorem 1, Lemma 3 has the corollary below:

Corollary 2 The timed transition system $G B_{2}$ has the following properties if $e \leq 1$ :

$$
\begin{aligned}
& \text { Property of } G B_{2} \quad \text { Property of } G B_{1} \\
& \left(R e q_{11}\right) \quad \ell \leq 30 \Rightarrow \int L e a k_{1} \leq 1+e \quad \text { Req of Leak in } G B_{1} \\
& \left(R e q_{21}\right), \ell \leq 30 \Rightarrow \int L e a k_{2} \leq 1+e \quad \text { Req of Leak in } G B_{1}
\end{aligned}
$$

It is interesting to notice that $R e q$ of the Gas Burner (which is already deduced from Corollary 1 in Theorem 2) can be also deduced from Corollary 2 and the valid formula

$$
\int\left(L e a k_{1} \vee L e a k_{2}\right)=\int L e a k_{1}+\int L e a k_{2}=2+2 e \leq 4
$$

\section{An implementation with flame failure}

When flame may disappear in the phase Burn, we can add another simple error recovery procedure which enters Idle from Burn by switching off the gas. Formally, we only have to redefine the action GoIdle in $G B_{2}$ as

$$
\begin{aligned}
\text { GoIdle } \stackrel{\text { def }}{=} & (\text { phase }=\text { Burn }) \wedge(\neg \text { Flame } \vee \neg \text { HeatReq }) \wedge\left(\text { phase } \text { f }^{\prime}=\text { Idle }\right) \\
& \wedge\left(\text { gas }^{\prime}=\text { off }\right) \wedge\left(\text { sensor }{ }^{\prime}=\text { off }\right)
\end{aligned}
$$

Let Leak $_{3}$ denote $($ phase $=$ Burn) $\wedge \neg$ Flame. The corresponding timed transition system $G B_{3}$ satisfies Corollary 2 and the following property

$$
\left(R e q_{31}\right): \quad \ell \leq 30 \Rightarrow \int L e a k_{3} \leq e
$$

which is of the same form as Req for the abstract state $L e a k$ in $G B_{1}$ and can be proved by the same proof routine by which $R e q$ is proved for $G B_{1}$.

Let $L e a k$ be the disjunction $L e a k_{1} \vee L e a k_{2} \vee L e a k_{3}$. By the valid formulas

$$
\begin{aligned}
\int L e a k & =\int\left(L e a k_{1} \vee L e a k_{2} \vee L e a k_{3}\right) \\
& =\int L e a k_{1}+\int L e a k_{2}+\int L e a k_{3}=2+3 e
\end{aligned}
$$


this gives the following correctness theorem for $G B_{3}$ :

Theorem 3 (Correctness of $G B_{3}$ ) Assume that $e \leq 2 / 3$, the transition system $G B_{3}$ satisfies the requirement Req of the Gas Burner system.

However, we should mention that $G B_{3}$ is not a refinement of $G B_{1}$ or $G B_{2}$. It does not implement $D e s$ as it does not meet $D e s_{2}$. In other words, we do not have a corollary for $G B_{3}$ corresponding to Corollary 1 or a theorem for $G B_{3}$ corresponding to Theorem 2.

\section{CONCLUSION}

We have proposed an approach to combine the DC approach and the TLTL approach to specification and verification of embedded real-time systems. The combination provides both the advantage of DC for directly modelling conventional dynamical systems with state that are functions of time, and the advantage of TTS for modelling computations in reactive systems. A central point is that the link between the approaches are formulated as syntactical rules

Within this framework, DC is used for specifying the system requirements and an initial design which intuitively corresponds to a simple TTS. Then we can refine (e.g. from $G B_{1}$ to $G B_{2}$ ) or adjust (e.g. from $G B_{2}$ to $G B_{3}$ ) the initial design within the TTS framework until we obtain an implementation which is a program.

The approach is illustrated by solving the Gas Burner example. The advantage of the combined approach becomes obvious if we compare with the solution in the single DC framework in (Ravn et al. 1993), and the solution in the single TLTL framework in (Lamport 1993): specifications of the system at different levels are simpler and better structured; the proofs of the correctness of the systems at different levels are easier as they directly reflect the structural induction of each step in the refinement/implementation, and concrete states (e.g. $L e a k_{1}, L e a k_{2}$ and $L e a k_{3}$ in $G B_{2}$ and $G B_{3}$ ) at a lower level are reasoned about in the same way as the corresponding abstract state (e.g. Leak in $G B_{1}$ ) at a higher level.

The combined logic allows us to describe multiple instantaneous transitions at a time point. For the same purpose, papers (Zhou \& Hansen 1996b) and (Xu 1997) introduce a dense-chop operator which can define the meaning of the sequential composition of state transitions. However, in both papers the intermediate states of a sequentially composite statement are hidden, and thus the semantics of $x:=1 ; x:=$ $x+2$ is the same as that of $x:=3$. This is a nice property if the approaches are used to deal with concurrent systems with no shared variables. However, in shared memory based models such as TTSs, the semantics of $(x:=x+1 ; x:=x+2) \|(x:=0)$ should be quite different from that of $x:=3 \| x:=0$; therefore we define DC over trajectories of a TTS.

Further work includes development of a full combined logic and extension of it to deal with hybrid transition systems (Manna \& Pnueli 1993).

Acknowledgements We wish to thank Xu Qiwen for the comments and discus- 
sions about the finite variability of a trajectory, and the anonymous referees for their comments.

\section{REFERENCES}

Abadi, M. \& Lamport, L. (1991). The existence of refinement mapping. Theoretical Computer Science 83(2), 253-284.

Abadi, M. \& Lamport, L. (1992). An old-fashioned recipe for real-time. In J. de Bakker, C. Huizing, W. de Rover \& G. Rozenberg, eds, Real-Time: Theory in Practice, Lecture Notes in Computer Science 600, Springer-Verlag, pp. 127.

Hansen, M. \& Zhou, C. (1997). Duration calculus: logical foundations. Formal Aspects of Computing 9(3), 283-330.

Henzinger, T., Manna, Z. \& Pnueli, A. (1994). Temporal proof methodologies for timed transition systems. Information and Computation 112(2), 273-337.

Keller, R. (1976). Formal verification of parallel programs. Communication of the ACM 19(7), 371-384.

Koymans, R., Vytopil, J. \& de Roever, W.-P. (1983). Real-time programming and asynchronous message passing. In Proc. 2nd Annual Symposium on Principles of Distributed Computing, ACM press, pp. 187-197.

Lamport, L. (1977). Proving the correctness of multiprocess programs. IEEE Transactions on Software Engineering 3(2), 125-143.

Lamport, L. (1993). Hybrid systems in $\mathrm{TLA}^{+}$. In R. Grossman, A. Nerode, A. Ravn \& H. Rischel, eds, Hybrid Systems, Lecture Notes in Computer Science 736, Springer-Verlag, pp. 77-102.

Lamport, L. (1994). The temporal logic of actions. ACM Transactions on Programming Languages and Systems 16(3), 872-923.

Manna, Z. \& Pnueli, A. (1981). The temporal framework for concurrent programs. In R. Boyer \& J. Moore, eds, The Correctness Problem in Computer Science, Academic Press, pp. 215-274.

Manna, Z. \& Pnueli, A. (1992). The Temporal Logic of Reactive and Concurrent Systems: Specification, Springer-Verlag.

Manna, Z. \& Pnueli, A. (1993). Verifying hybrid systems, In R. Grossman, A. Nerode, A. Ravn \& H. Rischel, eds, Hybrid Systems, Lecture Notes in Computer Science 736, Springer-Verlag, pp. 4-35.

Moszkowski, B. (1985). A temporal logic for multilevel reasoning about hardware. IEEE Computer 18(2), 10-19.

Ravn, A. \& Rischel, H. (1991). Requirements capture for embedded real-time systems. In Proc. IMACS-MCTS'91 Symposium on Modelling and Control of Technical Systems, Vol 2, pp. 1147-152.

Ravn, A., Rischel, H. \& Hansen, K. (1993). Specifying and verifying requirements of real-time systems. IEEE Transactions on Software Engineering 19(1), 4155.

Sørensen, M., Hansen, O. \& Løvengreen, H. (1994). Combining temporal specific- 
ation techniques. In Temporal Logic ICTL 94, Lecture Notes in Artificial Intelligence 827, Springer-Verlag, pp. 1-16.

Xu, Q. (1997). A semantics and verification of extended phase transition systems in duration calculus, In O. Maler, ed., International Workshop on Hybrid and Real-Time Systems, Lecture Notes in Computer Science 1201, SpringerVerlag, pp. 301-315.

Zhou, C. (1993). Duration calculi: an overview, In D. Bjørner, M. Broy \& I. Pottosin, eds, Proc. Formal Methods in Programming and Their Application, Lecture Notes in Computer Science 735, Springer-Verlag, pp. 256-266.

Zhou, C. \& Hansen, M. (1996a). An adequate first order interval logic. Technical Report 91, UNU/IIST, P.O. Box 3058, Macau.

Zhou, C. \& Hansen, M. (1996b). Chopping a point. In J. He, J. Cooke \& P. Wallis, eds, Proc. BCS FACS 7th Refinement Workshop: Theory and Practice of System Design.

Zhou, C., Hoare, C. \& Ravn, A. (1991). A calculus of durations. Information Processing Letters 40(5), 269-276.

Zhou, C., Ravn, A. \& Hansen, M. (1993). An extended duration calculus for hybrid real-time systems. In R. Grossman, A. Nerode, A. Ravn \& H. Rischel, eds, Hybrid Systems, Lecture Notes in Computer Science 736, Springer-Verlag, pp. 36-59.

\section{BIOGRAPHIES}

Zhiming Liu is a lecturer in Computer Science at the University of Leicester. He obtained his MSc in Computer Science from the Software Institute of the Chinese Academy of Sciences (Beijing, 1987) and Ph.D in Computer Science from the University of Warwick (UK, 1991). His research interest is in Theories of Distributed Real-Time Systems. He has worked and had long visits to the University of Warwick and the Technical University of Denmark.

Anders P. Ravn is a Reader at the Technical University of Denmark (DTU). He works on methods for engineering of embedded real-time systems based on coherent mathematical theories and techniques. He holds a MSc in Computer Science from the University of Copenhagen (1973) and is dr. techn from DTU (1995). He has worked in industry and had long visits to IBM T.J. Watson Research Center, Oxford University, and Kiel University.

Xiaoshan Li is an assistant professor of Software Engineering at University of Macau. He received his $\mathrm{PhD}$ degree at Institute of Software, the Chinese Academy of Sciences (Beijing, 1993). He worked in the University of Newcastle upon Tyne from October 1995 to March 1998. His research interests include formal methods in software engineering, hardware verification, specification of concurrent, real-time and hybrid systems. 